\title{
APLICABILIDADE DA REALIDADE VIRTUAL NA PROMOÇÃO DA SAÚDE E PREVENÇÃO DE DOENÇAS: ESTUDO DE REVISÃO
}

\author{
Mayara Alves Souza ${ }^{1}$, Drielly Lima Valle Folha Salvador ${ }^{2}$, Carlos \\ Alexandre Molena Fernandes ${ }^{3}$
}

\author{
${ }^{1}$ Acadêmica do Curso de Enfermagem, Universidade Estadual do Paraná Campus Paranavaí - UNESPAR. \\ alvessouzamayara@gmail.com \\ ${ }^{2}$ Enfermeira, Mestre em Enfermagem, Doutoranda do Programa de Pós-Graduação em Enfermagem, Universidade Estadual de \\ Maringá - UEM. enfdriellyvalle@gmail.com \\ ${ }^{3}$ Doutor em Ciências Farmacêuticas. Docente permanente do Programa de Pós-Graduação em Enfermagem, Universidade Estadual de \\ Maringá - UEM. carlosmolena126@gmail.com
}

\section{RESUMO}

Trata-se de um estudo de revisão integrativa desenvolvido em etapas cujo objetivo foi identificar, reunir e sintetizar informações extraídas de publicações científicas, acerca da aplicabilidade da realidade virtual em condições de saúde e doenças. A busca pelas publicações ocorreu entre julho e agosto de 2019, na base de dados - Scientific Electronic Library Online (SciELO). Foram analisados oito artigos científicos publicados em português e inglês, disponíveis na íntegra. Observou-se que a realidade virtual tem se revelado uma ferramenta eficaz no manejo da saúde, especialmente em condições e agravos que incluem déficits e traumas motores, como no acidente vascular encefálico e a paralisia cerebral. Também se mostrou eficaz no uso contra transtornos mentais comuns como ansiedade e medo e condições especiais como em casos de gestantes e crianças excepcionais.

PALAVRAS-CHAVE: Prevenção de doenças; Promoção da Saúde; Realidade Virtual.

\section{INTRODUÇÃO}

O uso da realidade virtual $(\mathrm{RV})$ no cuidado à saúde das pessoas pode ser um aliado como ferramenta tecnológica e existem evidências de que essa ferramenta pode promover benefícios como a melhora das funções motoras e cognitivas dos indivíduos, a interação social e maior satisfação e realização pessoal, por meio da utilização de recursos como fones de ouvido, óculos, luvas, computadores, videogames e sensores podem ser utilizados nessas intervenções (MAANI et al., 2011; WEISS et al.,2004).

A RV tem sido utilizada nas ciências da saúde com diferentes finalidades como na reabilitação de indivíduos, recuperação da saúde após lesões e traumas, na investigação, formação e educação em saúde e como intervenção terapêutica em condições específicas (LORENZO; BRACCIALLI; ARAÚJO, 2015).

Frequentemente, profissionais de saúde tem utilizado tecnologias em sua prática diária, porém, estudos que revisem e sintetizem as aplicações destas ferramentas e descreva quais foram as intervenções desenvolvidas, ainda são necessários para nortear aqueles que estão na prática clínica em diferentes âmbitos (POGATZKI-ZAHN; SEGELCKE; SCHUG, 2017).

Deste modo, este estudo tem como objetivo revisar a literatura científica disponível sobre a aplicabilidade da RV em diferentes contextos da prevenção de doenças e promoção da saúde.

\section{MATERIAIS E MÉTODOS}

Este estudo de revisão integrativa foi baseado no referencial de Mendes, Silveira e Galvão (2008), desenvolvido em etapas com o objetivo de reunir, compreender e sintetizar a temática da aplicabilidade da RV como estratégia para a promoção de saúde e prevenção de doenças. 
Para o desenvolvimento da pesquisa, procedeu-se a: A) definição do tema; B) estabelecimento dos critérios de inclusão; C) definição e seleção das informações a serem coletadas como desenho do estudo e condição e/ou doença que os autores visaram intervir, tipo de videogame e/ou console e tipo de jogo utilizado e, por fim, periódico da publicação e categoria profissional dos autores; D) interpretação dos resultados coletados; e E) síntese do conhecimento.

A coleta ocorreu entre os meses de julho e agosto de 2019 e, como estratégia de busca, foram utilizados os Descritores em Ciências da Saúde (DeCS): "Realidade Virtual" e "Saúde", nas bases de dados indexadas na SciELO - Scientific Electronic Library Online. Na sequência, foi realizada a leitura dos títulos e resumos a fim de identificar aqueles que se incluíam na temática. Por se tratar de estudo de revisão integrativa fica dispensado da aprovação do Comitê de Ética e Pesquisa com Seres Humanos.

\section{RESULTADOS E DISCUSSÃO}

Inicialmente a pesquisa pelos descritores retornou um total de 208 estudos. Destes, 111 foram excluídos por terem sido publicados há mais de cinco anos, 27 por não estarem publicados em língua portuguesa ou inglesa, 21 por não serem artigos originais e 55 por estarem repetidos e/ou não se enquadrarem no tema deste estudo (Figura 1).

\begin{tabular}{|c|c|c|c|c|c|c|c|c|}
\hline $\begin{array}{c}208 \\
\text { estudos }\end{array}$ & $\Rightarrow$ & $\begin{array}{l}111 \text { fora } \\
\text { do limite } \\
\text { temporal }\end{array}$ & $\Rightarrow$ & $\begin{array}{l}21 \text { artigos } \\
\text { não } \\
\text { originais }\end{array}$ & $\Rightarrow$ & $\begin{array}{c}55 \text { artigos } \\
\text { repetidos ou fora } \\
\text { do tema em } \\
\text { estudo }\end{array}$ & $\Rightarrow$ & $\begin{array}{l}\text { Total = } 8 \text { artigos } \\
\text { selecionados para } \\
\text { análise completa }\end{array}$ \\
\hline
\end{tabular}

Figura 1: Fluxograma do processo de seleção dos artigos utilizados na revisão $(n=8)$.

Fonte: Dados da pesquisa

As características metodológicas dos estudos analisados estão apresentadas na Tabela 1.

Tabela 1: Caracterização metodológica dos artigos científicos segundo autoria, ano de publicação, periódico, desenho do estudo e tamanho da amostra $(n=8)$.

\begin{tabular}{lcccc}
\hline No & AUTORIA/ANO & PERIÓDICO & $\begin{array}{c}\text { DESENHO } \\
\text { DO ESTUDO }\end{array}$ & N \\
\hline 01 & Arnoni et al. (2019) & Fisioterapia e Pesquisa & Longitudinal & 8 \\
\hline 02 & Zacarin, Borloti e Haydu (2019) & Tendências em Psicologia & Transversal & 6 \\
\hline 03 & Gonçalves et al. (2018) & Arquivos de Neuro-Psiquiatria & Estudo clínico & 18 \\
\hline 04 & Ribeiro, Sousa e Viana (2017) & Fisioterapia em Movimento & Ensaio Clinico & 32 \\
\hline 05 & Carneiro et al. (2016) & Fisioterapia em Movimento & Descritivo & 27 \\
\hline 06 & Silva e Iwabe-Marchese (2015) & Fisioterapia e Pesquisa & Descritivo & 1 \\
\hline 07 & Menezes et al. (2015) & MedicalExpress & Transversal & 30 \\
\hline 08 & Lorenzo, Braccialli e Araújo (2015) & Revista Brasileira de Educação Especial & Descritivo & 1 \\
\hline
\end{tabular}

Fonte: Dados da pesquisa

A análise metodológica dos estudos em questão, permite perceber que apenas o estudo de Silva e Iwabe-Marchese (2015) relatou qual era a categoria profissional dos autores, neste caso, fisioterapeutas. Os demais estudos não informam claramente. Porém, todos os autores dos estudos analisados possuíam vínculo com alguma universidade. Tal fato se releva importante, visto que este vínculo está atrelado ao acesso a conteúdo científico e atualizado. 
Tabela 2: Caracterização dos artigos científicos segundo autoria, ano de publicação, tamanho e idade da amostra, doença/condição a ser estudada, ferramenta tecnológica utilizada e tipo de jogo $(n=8)$.

\begin{tabular}{|c|c|c|c|c|c|c|}
\hline AUTORIA/ANO & IDADE & $\mathbf{N}$ & SEXO & $\begin{array}{l}\text { DOENÇA/ } \\
\text { CONDIÇÃO }\end{array}$ & $\begin{array}{l}\text { VIDEOGAME/ } \\
\text { CONSOLE }\end{array}$ & JOGO \\
\hline Arnoni et al. (2019) & $\begin{array}{l}5 \text { a } 14 \\
\text { anos }\end{array}$ & 8 & $\mathrm{~F} / \mathrm{M}$ & Paralisia Cerebral & $\begin{array}{c}\text { Xbox } 360+ \\
\text { Kinect@ }\end{array}$ & Não citado \\
\hline $\begin{array}{c}\text { Zacarin, Borloti e } \\
\text { Haydu (2019) }\end{array}$ & $\begin{array}{c}18 \text { a } 26 \\
\text { anos }\end{array}$ & 6 & $\mathrm{~F}$ & $\begin{array}{l}\text { Transtorno de } \\
\text { Ansiedade }\end{array}$ & Simulador & Não citado \\
\hline $\begin{array}{c}\text { Gonçalves et al. } \\
\text { (2018) }\end{array}$ & $\begin{array}{l}\text { Maiores de } \\
18 \text { anos }\end{array}$ & 18 & $\mathrm{~F} / \mathrm{M}$ & AVE & Nintendo Wiiß & $\begin{array}{c}\text { Wii Sports } \\
\text { Tennis e Wii } \\
\text { Sports Bowling }\end{array}$ \\
\hline $\begin{array}{l}\text { Ribeiro, Sousa e } \\
\text { Viana (2017) }\end{array}$ & $\begin{array}{l}18 \text { a } 35 \\
\text { anos }\end{array}$ & 32 & $\mathrm{~F}$ & $\begin{array}{l}\text { Equilibrio Postural } \\
\text { de Gestantes }\end{array}$ & Nintendo Wiiß & WiiFit Plus ${ }^{\circledR}$ \\
\hline $\begin{array}{l}\text { Carneiro et al. } \\
\quad(2016)\end{array}$ & $\begin{array}{l}30 \text { a } 80 \\
\text { anos }\end{array}$ & 27 & $\mathrm{~F} / \mathrm{M}$ & AVE & $\begin{array}{l}\text { Notebook, } \\
\text { projetor, } \\
\text { Kinect@ }\end{array}$ & IKAPP \\
\hline $\begin{array}{c}\text { Silva e Iwabe- } \\
\text { Marchese (2015) }\end{array}$ & 12 anos & 1 & M & $\begin{array}{c}\text { Paralisia Cerebral } \\
\text { Atáxica }\end{array}$ & Nintendo $\mathrm{Wii} B$ & WiiFit Plus® \\
\hline $\begin{array}{c}\text { Menezes et al. } \\
(2015)\end{array}$ & $\begin{array}{c}10 \text { a } 34 \\
\text { anos }\end{array}$ & 30 & $\mathrm{~F} / \mathrm{M}$ & $\begin{array}{c}\text { Síndrome de } \\
\text { Down }\end{array}$ & Smartphone & $\begin{array}{c}\text { Marble } \\
\text { Maze Classic } \AA\end{array}$ \\
\hline $\begin{array}{l}\text { Lorenzo, Braccialli } \\
\text { e Araújo (2015) }\end{array}$ & $\begin{array}{l}10 \text { anos e } \\
5 \text { meses }\end{array}$ & 1 & M & $\begin{array}{c}\text { Síndrome de } \\
\text { Down }\end{array}$ & $\begin{array}{l}\text { Xbox } 360+ \\
\text { Kinect } \AA\end{array}$ & Não citado \\
\hline
\end{tabular}

Legenda: N: tamanho da amostra, F: feminino, M: masculino, AVE: acidente vascular encefálico.

Fonte: Dados da pesquisa

A análise da Tabela 2, permite observar que a aplicação da RV como intervenção em portadores de doenças como o acidente vascular encefálico, evidencia que a RV pode ser uma ferramenta importante na reabilitação e recuperação da saúde das pessoas. Tal informação vai de encontro com outros estudos que mostraram que essa ferramenta pode auxiliar no processo de treinamento cognitivo e motor, tornando a recuperação mais rápida e eficaz (VAN TWILLERT; BREMER; FABER, 2007).

A idade dos participantes foi diversificada. Diferentes públicos foram estudados em diferentes condições de saúde. Esse fator se revela especialmente importante, visto que Freitas e Spadoni (2019), em estudo sobre avanços médicos, evidenciaram que a heterogeneidade de grupos e a singularidade de cada ser humano, fornece aos pesquisadores diferentes indicadores sobre a efetividade das ferramentas tecnológicas. Porém, também se faz necessário o desenvolvimento de pesquisas padronizadas para definição da eficácia destas ferramentas.

\section{CONCLUSÃO}

Evidenciou-se que as ferramentas tecnológicas e o uso da realidade virtual tem sido empregado com sucesso em diferentes condições de saúde e de agravos. Portadores de doenças agudas e crônicas têm se beneficiado com o a realidade virtual como intervenção.

Sua aplicabilidade tem se mostrado viável especialmente em condições que envolvem comprometimentos motores e cognitivos, decorrentes de complicações fisiológicas. Profissionais de saúde e pesquisadores devem ser encorajados a aplicarem a realidade virtual em diferentes ambientes e condições de saúde e doença dos indivíduos e a desenvolverem novos estudos que analisem e compartilhem as possiblidades das tecnologias no âmbito da saúde.

\section{REFERÊNCIAS}


ARNONI, J. L. B. et al. Efeito da intervenção com videogame ativo sobre o autoconceito, equilíbrio, desempenho motor e sucesso adaptativo de crianças com paralisia cerebral: estudo preliminar. Fisioterapia e Pesquisa, [s.I.], v. 25, n. 3, p.294-302, set. 2018.

CARNEIRO, M. I. S. et al. Applicability of a motor rehabilitation system in stroke victims. Fisioterapia em Movimento, [s.I.], v. 29, n. 4, p.723-730, dez. 2016. FapUNIFESP (SciELO).

GONÇALVES, M. G. et al. Effects of virtual reality therapy on upper limb function after stroke and the role of neuroimaging as a predictor of a better response. Arquivos de Neuropsiquiatria, [s.I.], v. 76, n. 10, p.654-662, out. 2018.

AS, D. M. O.; SPADONI, V. S. Is virtual reality useful for pain management in patients who undergo medical procedures? Einstein (são Paulo), [s.l.], v. 17, n. 2, p.1-3, 2019.

LORENZO, S. M.; BRACCIALLI, L. M. P.; ARAUJO, R. C. T. Realidade Virtual como Intervenção na Síndrome de Down: uma Perspectiva de Ação na Interface Saúde e Educação. Revista Brasileira de Educação Especial, [s.l.], v. 21, n. 2, p.259-274, jun. 2015.

MAANI, C. V. et al. Virtual Reality Pain Control During Burn Wound Debridement of CombatRelated Burn Injuries Using Robot-Like Arm Mounted VR Goggles. The Journal Of Trauma: Injury, Infection, and Critical Care, [s.I.], v. 71, n.1, p.125-130, jul. 2011.

MENDES, K.S.; SILVEIRA, R.C.C.P.; GALVÃO, C.M. Revisão integrativa: método de pesquisa para a incorporação de evidências na saúde e na enfermagem. Texto \& Contexto - Enfermagem, v. 17, n. 4, p.758-764, dez. 2008.

MENEZES, L. C. de et al. Motor learning in mobile (cell phone) device in Down syndrome patients - pilot project. Medical Express, v. 2, n. 4, p.1-5, 2015.

POGATZKI-ZAHN, E. M.; SEGELCKE, D.; SCHUG, S. A.. Postoperative pain-from mechanisms to treatment. Pain Reports, [s.l.], v. 2, n. 2, p.588-1, 2017.

RIBEIRO, S. O.; SOUSA, V. P. S.; VIANA, E. S. R. Influence of virtual reality on postural balance and quality of life of pregnant women: controlled clinical trial randomized. Fisioterapia em Movimento, v. 30, n. 1, p.111-120, 2017.

SILVA, R. R.; IWABE-MARCHESE, C. Uso da realidade virtual na reabilitação motora de uma criança com Paralisia Cerebral Atáxica: estudo de caso. Fisioterapia e Pesquisa, São Paulo, v. 22, n. 1, p.97-102, mar. 2015.

ZACARIN, M. R. J.; BORLOTI, E.; HAYDU, V. B. Behavioral Therapy and Virtual Reality Exposure for Public Speaking Anxiety. Temas em Psicologia, v. 27, n. 2, p.491-507, 2019. Associacao Brasileira de Psicologia.

WEISS, P. L. et al. Video capture virtual reality as a flexible and effective rehabilitation tool. Journal Of Neuroengineering And Rehabilitation, v. 1, n. 1, p.12-12, 2004. Springer Nature. http://dx.doi.org/10.1186/1743-0003-1-12. 